\title{
Société de médecine de Bâle
}

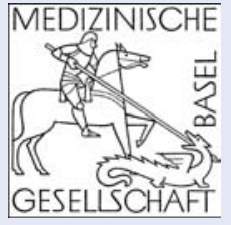

\author{
La Société de médecine de Bâle (MedGes) est l'organisation professionnelle \\ de tous les médecins exerçant en ville de Bâle, indépendamment de leurs \\ tâches et positions. Elle constitue I'organisation de base FMH du canton \\ de Bâle-Ville.
}

\section{Historique}

La MedGes a été officiellement fondée le 15 novembre 1860 en mettant fin à une série d'échecs dans la tentative de se regrouper en une association. Plusieurs médecins s'étaient réunis dès mars 1860 pour discuter des problèmes survenant dans leur activité au cabinet médical. Ces médecins se sont ensuite rencontrés une fois par mois pour cultiver essentiellement la convivialité. Mais comme le besoin de se consacrer à une activité scientifique avait crû au fil du temps, on mit sur pied une commission chargée d'élaborer un projet de statuts pour une nouvelle société de médecine. A la première séance tenue le 15 novembre 1860, les 18 membres présents examinèrent les statuts et procédèrent à des élections, en fondant du même coup la MedGes.
\end{abstract}

\section{Organisation et tâches}

La MedGes est organisée sous forme d'association. Ses tâches sont réparties en sept domaines, chacun se trouvant sous la responsabilité d'un membre du comité. Ces domaines sont les suivants: présidence, assurances et tarifs, formation continue, prestations de services, administration des membres, communication, finances. Depuis l'introduction du TARMED, un temps énorme a été investi dans les négociations tarifaires. La MedGes a dû faire face à des difficultés les plus diverses dans ce contexte. La caractéristique de centre médical, propre à la Ville de Bâle, d'une part, et les données démographiques et socioéconomiques spéciales d'un canton-ville, d'autre part, lui ont permis de creuser en profondeur la systématique relative à l'application tarifaire.

La communication interne et extérieure a également été intensifiée. La MedGes tient à exercer une influence sur la politique de la santé au niveau cantonal (et régional) et à être considérée comme un partenaire compétent. Elle entretient des contacts étroits avec les autres prestataires de la santé publique.

\section{Centrale médicale d'appel d'urgence MNZ! 06126115 15: une prestation de la MedGes}

En juin 1965, la MedGes a fondé la centrale médicale d'appel d'urgence MNZ! 06126115 15, devenue aujourd'hui une prestation au service de toute la région et dont la population ne pourrait plus se passer. Depuis 40 ans, 24 heures sur 24 et 365 jours l'an, la MNZ! 0612611515 organise le service de garde à Bâle et les environs, sans interruption et de manière fiable; elle répond aux personnes de Bâle-Ville, Bâle-Campagne et certaines parties des cantons d'Argovie et de Soleure, confrontées à des problèmes de santé subits. Selon la situation, la centrale leur donne directement des conseils ou organise les secours nécessaires. Elle offre son aide pour trouver un médecin de famille ou un dentiste ou (s'il n'est pas atteignable) le médecin ou le dentiste de garde; elle organise le transport ambulancier et les soins à domicile, donne des renseignements sur le service de piquet pour la privation de liberté à des fins préventives, vend et s'occupe de la maintenance des systèmes d'alarme de la Croix-Rouge suisse et conserve les dispositions de fin de vie des patients. Les appels sont gratuits (c'est-à-dire tarifs téléphoniques normaux sans taxe supplémentaire). Le nombre d'appels se sont élevés ces dernières années à environ 80000 par année. La centrale MNZ! 0612611515 est cofinancée, sous forme de contributions ou de subsides, par la Société de médecine de Bâle-Ville, la Société médicale de Bâle-Campagne et certaines organisations partenaires de même que par le canton de Bâle-Ville et les communes de Bâle-Campagne. 